\title{
Solar wind and geomagnetism: toward a standard classification of geomagnetic activity from 1868 to 2009
}

\author{
J. L. Zerbo ${ }^{1,2}$, C. Amory Mazaudier ${ }^{2}$, F. Ouattara ${ }^{3}$, and J. D. Richardson ${ }^{4}$ \\ ${ }^{1}$ Université Polytechnique de Bobo Dioulasso, 01 BP 1091 Bobo-Dioulasso 01, Burkina Faso \\ ${ }^{2}$ LPP-Laboratoire de Physique des Plasmas/UPMC/Polytechnique/CNRS, UMR 7648, 4 Avenue de Neptune, \\ 94107 Saint-Maur-des-Fossés, France \\ ${ }^{3}$ Ecole Normale Supérieure de 1'Université de Koudougou, BP 376 Koudougou, Burkina Faso \\ ${ }^{4}$ Center for Space Research, M.I.T., Cambridge, USA \\ Correspondence to: C. Amory Mazaudier (christine.amory@1pp.polytechnique.fr)
}

Received: 10 October 2011 - Revised: 9 February 2012 - Accepted: 22 February 2012 - Published: 27 February 2012

\begin{abstract}
We examined solar activity with a large series of geomagnetic data from 1868 to 2009 . We have revisited the geomagnetic activity classification scheme of Legrand and Simon (1989) and improve their scheme by lowering the minimum Aa index value for shock and recurrent activity from 40 to $20 \mathrm{nT}$. This improved scheme allows us to clearly classify about $80 \%$ of the geomagnetic activity in this time period instead of only $60 \%$ for the previous Legrand and $\mathrm{Si}$ mon classification.
\end{abstract}

Keywords. Geomagnetism and paleomagnetism (Time variations, secular and long term)

\section{Introduction}

Geomagnetic activity may be defined as the magnetosphere's response to the transitory variation of solar activity. Legrand and Simon (1985, 1989) and Simon and Legrand (1989) show the existence of two categories of magnetic perturbations: the first is organized into recurrent features and the second forms a range of intense and short events distributed at random. These authors divide geomagnetic activity into four classes: quiet, recurrent, shock, and fluctuating (Legrand and Simon, 1989; Richardson et al., 2000; Richardson and Cane, 2002; Ouattara et al., 2009; Ouattara and Amory Mazaudier, 2009).

To determine the classes, Legrand and Simon (1989) and Simon and Legrand (1989) built a diagram similar to Bartels 27-days rotation using the geomagnetic index Aa from 1868 to 1977 . This diagram, named a pixel diagram, represents the geomagnetic data as a function of solar activity for each solar rotation (27 days) and gives an overview of the geoeffectiveness of solar events.

Of these four classes of geomagnetic activity, only three classes (quiet activity, recurrent activity and shock activity) are clearly selected and defined. The fourth (fluctuating) contains all data which does not fit into the other three categories.

In light of the strong correlation between the Aa index and solar wind established by Svalgaard (1977) and the fact that $91.5 \%$ of solar activity is reflected by solar wind speed (Legrand and Simon, 1985, 1989), we decided to try to further refine fluctuating activity to better understand its origins. We find that it is appropriate to subdivide the fluctuating activity into three new classes including Coronal Mass Ejection (CME) manifestations and moderate solar wind effects (Gopalswamy et al., 2003; Ramesh, 2010).

\section{Data and analysis}

\subsection{Data}

The times of sudden storm commencements (SSC), which are rapid increases in the magnetic field observed at ground, and the number of CMEs per days are taken from http: //isgi.latmos.ipsl.fr/ and http://cdaw.gsfc.nasa.gov/CME_list, respectively. The solar wind speed and the international sunspot number (SSN) are obtained from http://omniweb. gsfc.nasa.gov/form/dx1.html. The SSN data are used to determine solar cycle phases. 

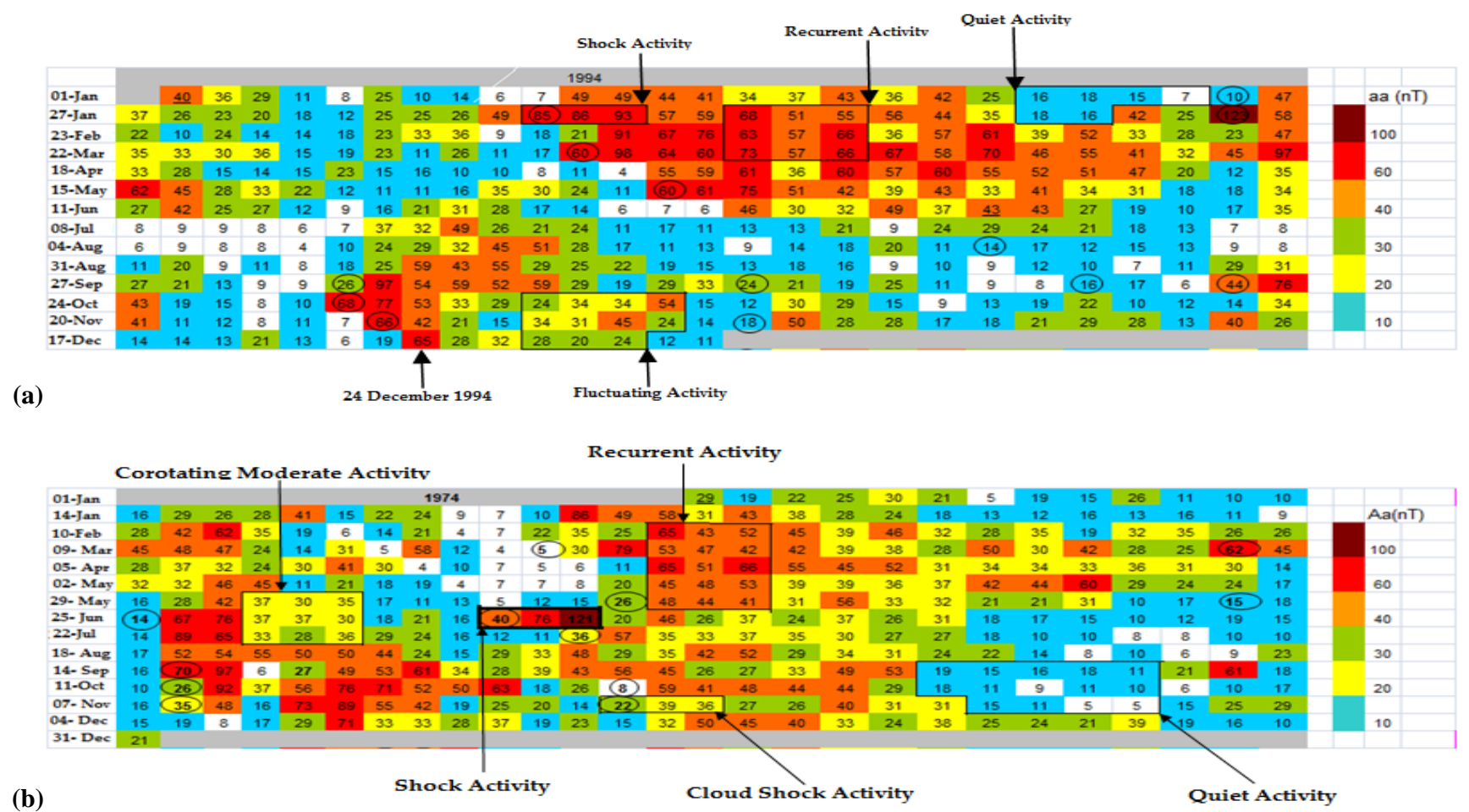

(b)

Shock Activity

Cloud Shock Activity

Quiet Activity

Fig. 1. Pixel diagrams for years 1994 (a) and 1974 (b).

\subsection{Data analysis}

To classify geomagnetic activity, Legrand and Simon (1989) use the following criteria: (1) SSC times are used to determine when shock events contribute to geomagnetic activity, (2) $91.5 \%$ of solar activity is reflected by solar wind speed, (3) the strong correlation between Aa (Mayaud, 1971, 1973, 1980) and the solar wind speed (Svalgaard, 1977). They divided the observations into four classes of geomagnetic activity: Shock activity (SA), quiet activity (QA), recurrent activity (RA) and fluctuating activity (FA), illustrated in Fig. 1a. These four classes are fully described in Legrand and Simon (1989) and Ouattara and Amory-Mazaudier (2009). It is, however, important to note that Legrand and Simon (1985) only classified $43 \%$ of SSCs as shock events, including only those on days with $\mathrm{Aa}>40 \mathrm{nT}$. The remainders were classified as fluctuating activity $\left(V \geq 450 \mathrm{~km} \mathrm{~s}^{-1}\right)$. Similarly, they include in RA only times when Aa is greater than $40 \mathrm{nT}$.

To refine the fluctuating activity (Legrand and Simon, 1989), as suggested in introduction, we use a 142 year-long Bartels diagram of Aa indices, SSC reports and two new classes with the following criteria:

(4a) Corotating Moderate Activity. This category of geomagnetic activity is defined as corotating, stable solar wind stream producing moderate geomagnetic effects, Aa between $20 \mathrm{nT}$ and $40 \mathrm{nT}$. We identify these events in the pixel diagram (Fig. 1) by the recurrence from on solar rotation to the next of yellow and green areas with no SSCs. (4b) Cloud Shock Activity. These events are shocks which cause only a moderate increase of activity level. We select only pixels within three days after SSCs with the colours yellow, green in the pixel diagram (Bartels diagram), and so the Aa indices are between $20 \mathrm{nT}$ and $40 \mathrm{nT}$.

(4c) Unclear Activity. This transient activity is composed of days not included in any other classes. Its annual level is obtained after subtracting Cloud Shock Activity and Corotating Moderate Activity annual levels from the fluctuating activity defined by Legrand and Simon (1989). Thus, we have increased from four to six the number of geomagnetic activity classes. Figure 1a is an example of pixel diagram for year 1994 which illustrates the five clear activity classes: quiet, recurrent, shock (defined by Legrand and Simon, 1989), cloud shock and corotating (defined in this paper).

In the next paragraphs we adopted the following notations: (QA) for Quiet Activity, (SA) for Shock Activity, (RA) for Recurrent Activity, (CSA) Cloud Shock Activity and (CMA) Corotating Moderate Wind Activity. The Unclear Activity due to the oscillation of the neutral sheet will be noted (UA).

For each year, the geomagnetic activity level characterized by the Aa indices can be expressed now by the sum of the six classes of geomagnetic activity: 
(a)

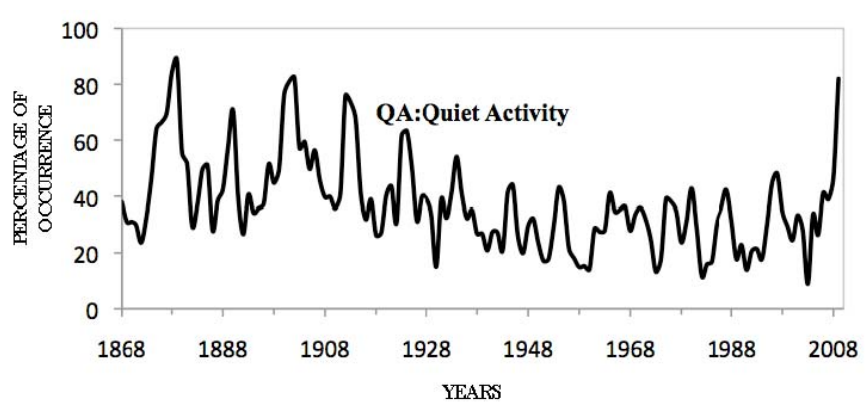

(c)

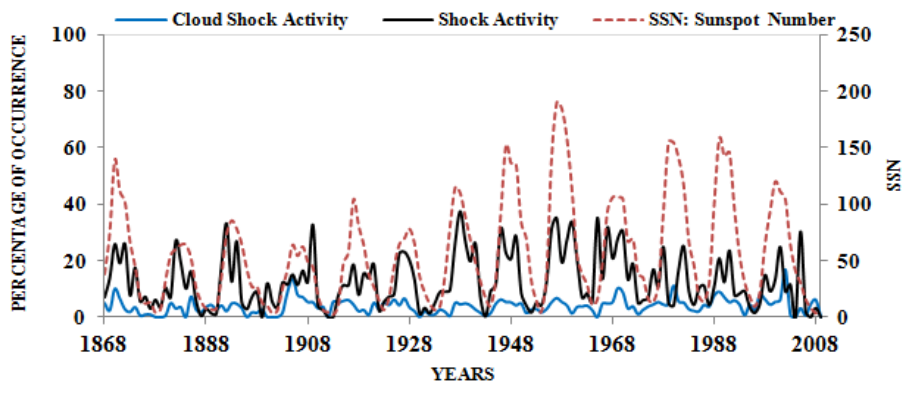

(b)

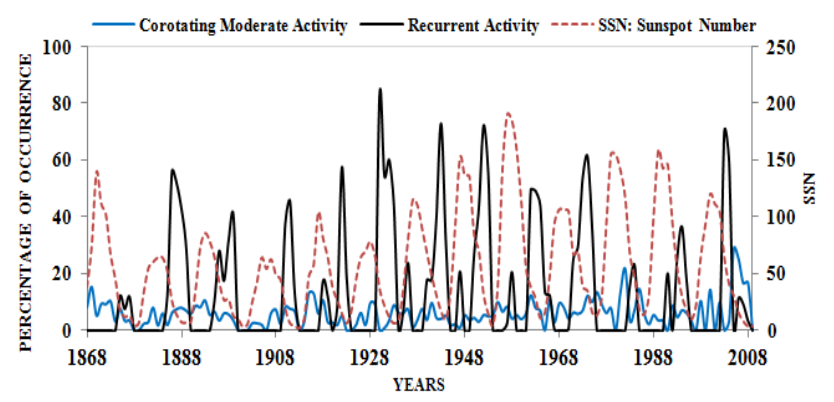

(d)

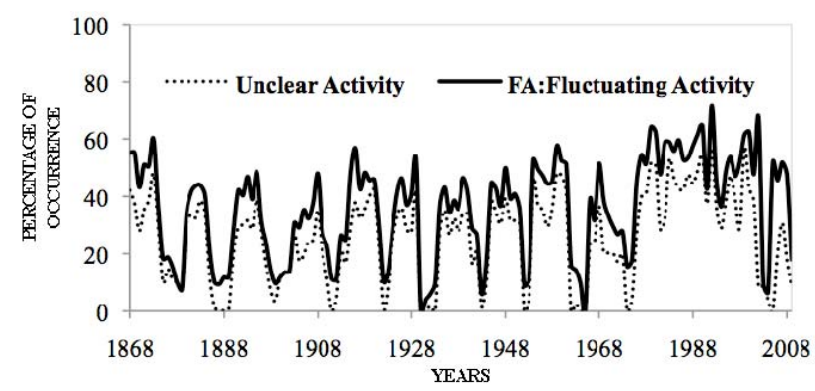

Fig. 2. Levels of quiet activity (a), recurrent activity, corotating moderate activity, and sunspot number levels (b), shock activity, cloud shock activity, and sunspot number levels (c), fluctuating activity and unclear activity levels (d) from 1868 to 2009.

Geomagnetic activity $=\mathrm{QA}+\mathrm{SA}+\mathrm{RA}+\mathrm{CSA}+\mathrm{CMA}+\mathrm{UA}(1)$

By the past with Simon and Legrand (1989), the geomagnetic activity was the sum of four classes of activity:

Geomagnetic activity $=\mathrm{QA}+\mathrm{SA}+\mathrm{RA}+\mathrm{FA}$;

with $\mathrm{FA}=\mathrm{CSA}+\mathrm{CMA}+\mathrm{UA}$.

For each class we determine the yearly level of activity as the sum of all the daily Aa of the days of the class.

For example the QA activity level of a year is given by:

$\mathrm{QA}=\sum_{i=1}^{N} \mathrm{Aa}$ (Quiet day)

$N$ : number of quiet days in the year.

\section{Results}

For the 142 years covered by this study, we divided the fluctuating activity into three news classes (corotating activity, cloud shock activity and unclear transient activity) to obtain six classes of geomagnetic activity. Figure 2 plots the yearly levels of the different classes of geomagnetic activity and the sunspot number from 1868 to 2009 . Figure 2a shows that quiet magnetic days are more prevalent from the end of the 1800s to $\sim 1950$ and at the end of the sunspot cycle 23 (1996-2009) and that the distribution is relatively flat during the second half of the 1900 s. Figure $2 b$ superimposes the recurrent activity (Legrand and Simon, 1989), the corotating moderate activity and the sunspot number. The higher magnetic activity (RA) dominated the CMA activity during most of the solar cycles from 1868 to 2009. Figure 2c deals with shock activity,cloud shock activity and the sunspot number long time evolution. The lowest shock activity levels were observed during cycles 15 (1913-1923) and 23 (19962009) while cloud shock activity reaches its highest level in the same time periods. Figure $2 \mathrm{~d}$ superimposes the long time variations of fluctuating activity (Legrand and Simon, 1989) and unclear activity. The difference in these two curves is due to the removal of CSA and CMA from fluctuating activity, which will be discussed later. Figure $2 \mathrm{c}$ superimposes the yearly sunspot number on the percentages of shock activity and cloud shock activity from 1868 to 2009 . We can see a close link between sunspot number and both activity levels time evolution. Figure $2 b$ shows that the largest percentage of recurrent activity occurs after the maximum in the sunspot number. This figure also shows that significant corotating activity is observed near sunspot maximum but that the largest percentage occurs in the declining phase. Figure $3 a$ and $b$ superimposes the sunspot number on the percentage of shock events and recurrent events, respectively. Figure $3 \mathrm{a}$ indicates that the highest contribution of shocks occurs near solar maximum. Figure $3 \mathrm{~b}$ shows that the most important contributions from high-speed solar wind streams are observed in the declining phase of sunspot cycle. 
Table 1. Components of the cycle of the geomagnetic activity, Legrand and Simon (1989).

\begin{tabular}{|c|c|c|c|}
\hline & Identification in Bartels diagrams & $\begin{array}{l}\text { Distribution among the phase of } \\
\text { the sunspot cycle }\end{array}$ & $\begin{array}{l}\text { Relationship with sunspot cycle } \\
\text { activity }\end{array}$ \\
\hline Quiet days & Recurrent patterns of aa $<20 \mathrm{nT}$. & $\begin{array}{l}\text { A nearly constant annual level } \\
\sim 6 \mathrm{nT} \\
\text { A peak in the annual quiet day } \\
\text { number at } m \text { or } m+1\end{array}$ & $\begin{array}{l}\text { Both the sum (from } m-4 \text { to } \\
M+3 \text { ) and the peak of the quiet } \\
\text { day numbers are inversely pro- } \\
\text { portional to } R \max (M)\end{array}$ \\
\hline Fluctuating activity & $\begin{array}{l}\text { Recurrent patterns of disturbed } \\
\text { days with a fluctuating level at the } \\
\text { time scales of day and of rotation }\end{array}$ & From $m$ or $m+1$ to $M+3$ or later & $\begin{array}{l}\text { Sum( during the full cycle) } \\
\text { closely correlated to } R \max (M)\end{array}$ \\
\hline \multirow{2}{*}{$\begin{array}{l}\text { Recurrent storms } \\
\text { (high speed wind } \\
\text { stream activity) }\end{array}$} & $\begin{array}{l}\text { Smooth high intensity recurrence } \\
\text { patterns of more than } 3 \text { rotations }\end{array}$ & $\begin{array}{l}\text { With equinoctial properties from } \\
M+3 \text { to } m \text { or } m+1\end{array}$ & $\begin{array}{l}\text { Sum (during a } 4 \mathrm{yr} \text { interval) } \\
\text { closely correlated to } R \max (M)\end{array}$ \\
\hline & & $\begin{array}{l}\text { Without any seasonal property } \\
\text { from } M+1 \text { to } m-2\end{array}$ & $\begin{array}{l}\text { Not any link at all with any } \\
\text { sunspot activity }\end{array}$ \\
\hline $\begin{array}{l}\text { SSC-Storm（shock } \\
\text { event activity） }\end{array}$ & $\begin{array}{l}\text { A series of definite SSC-Storm of } \\
\text { short duration ( } 2-3 \text { days) without } \\
\text { any recurrence property }\end{array}$ & $\begin{array}{l}\text { Events occurring in any phase Se- } \\
\text { vere storm }(\text { aa }<100 \mathrm{nT}) \text { grouped } \\
\text { from } M-1 \text { to } M+4 \text { for } 88 \% \text { of } \\
\text { them }\end{array}$ & $\begin{array}{l}\text { "Loose" correlation, if any, of the } \\
\text { sum( during a cycle) with } R \max \end{array}$ \\
\hline
\end{tabular}

Table 2. Correlation coefficients (SA, CSA) and (RA, CMA) for cycle 11 to cycle 23 .

\begin{tabular}{ccc}
\hline $\begin{array}{c}\text { Solar } \\
\text { cycle }\end{array}$ & $\begin{array}{c}\text { Correlation coefficient } \\
\text { (SA, CSA)/yearly } \\
\text { level of activity }\end{array}$ & $\begin{array}{c}\text { Correlation coefficient } \\
\text { (RA, CMA)/yearly } \\
\text { level of activity }\end{array}$ \\
\hline 11 & 0.93 & 0.86 \\
12 & 0.82 & 0.85 \\
13 & 0.83 & 0.74 \\
14 & 0.81 & 0.94 \\
15 & 0.80 & 0.82 \\
16 & 0.87 & 0.78 \\
17 & 0.89 & 0.70 \\
18 & 0.82 & 0.76 \\
19 & 0.89 & 0.77 \\
20 & 0.84 & 0.75 \\
21 & 0.80 & 0.89 \\
22 & 0.79 & 0.93 \\
23 & 0.79 & 0.72 \\
\hline
\end{tabular}

\section{Discussion and conclusion}

We investigate geomagnetic activity and its solar wind origins from 1868 to 2009 (142 years). We define three news classes of geomagnetic activity from the fluctuating activity class of Legrand and Simon (1989). Thus we now define six classes of geomagnetic activity, quiet activity (QA), recurrent activity (RA), shock activity (SA), cloud shock activity (CSA), corotating moderate activity (CMA) and unclear activity (UA). CSA, CMA and UA are extracted from the fluctuating activity previously defined by Legrand and Simon
Table 3. Comparison between the geomagnetic classification (Legrand and Simon criterion) and the classification after the refinement of the fluctuating activity.

\begin{tabular}{lcc}
\hline & $\begin{array}{c}\text { Legrand and } \\
\text { Simon (1989) }\end{array}$ & $\begin{array}{c}\text { New classification } \\
\text { Extension of RA and SA }\end{array}$ \\
\hline Quiet Activity & $34 \%$ & $34 \%$ \\
Recurrent Activity & $14 \%$ & $26 \%$ \\
Shock Activity & $13 \%$ & $19 \%$ \\
Fluctuating/unclear Activity & $39 \%$ & $21 \%$ \\
\hline
\end{tabular}

(1989). The new classification allows us to assign about $80 \%$ of the days into specific geomagnetic actitivy classes (as opposed to the FA and UA classes which are composed of days which fit not other group) in comparison to $60 \%$ assigned with the previous scheme (Legrand and Simon, 1989). From Fig. 2c, it is shown the strong correlation between cloud shock activity level and sunspot number variations.

The concordance between the cloud shock activity and the shock activity time evolutions throughout the many sunspot cycles suggests that both classes have the same mechanism. The different levels of Aa index probably result from differences in shock strength due to differences in the CMEs on the Sun which generate these shocks. We also point out a good correlation $(\geq 0.79)$ between the yearly level of SA and CSA for cycle 11 to cycle 23 as summarized in Table 2. Thus SA and CSA are different parts of a distribution formed from the same type of event and could be combined into one class. Figure 3a shows the sum of shock activity and cloud shock activity levels from 1868 to 2009. This figure points out a fairly constant in shock event levels was present for all the 
(a)

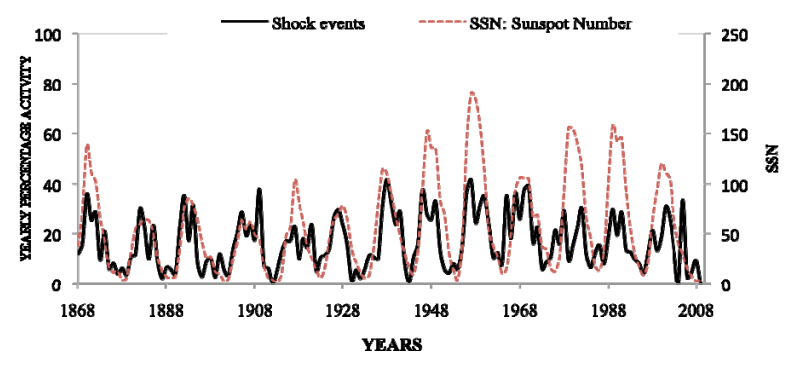

(b)

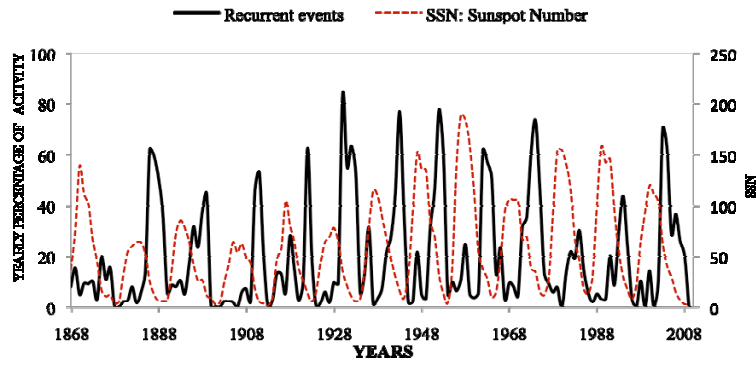

Fig. 3. levels of the shock events and the sunspot number (a), levels of the recurrent events and the sunspot number (b).

solar cycles from 1868 to 2009 in contrary to the time variations of SA and CSA plotted separately in Fig. 2c.

Table 1, taken from Legrand and Simon (1989), summarizes their classification scheme and the variation of each class with the sunspot cycle. The recurrent events reach their peak a few years before the sunspot minimum and sometimes near the sunspot maximum. Figure $2 b$ shows that corotating moderate activity which reaches its peak near the sunspot maximum or few years before sunspot minimum (sunspot declining phase) is in concordance with recurrent events occurrence summarized in Table 1. Sunspot maximum and the declining phase are dominated by recurrent high wind stream flowing from coronal holes. In addition, the yearly level of RA and CMA present significant correlation coefficient $(\geq 0.70)$ for each solar cycle since cycle 11 as shown in Table 2. Thus it appears that CMA and RA are also generated by the same mechanism. These results express the necessity to combine CMA and RA to keep one class as we did for SA and CSA. Figure $3 b$ gives an overview of the recurrent events (CMA plus RA) and the sunspot number from 1868 to 2009 . The recurrent events are likely governed by the distribution of coronal hole high-speed solar wind which occurs most often at low-latitudes in the declining phase of the sunspot cycle. The Table 3 summarizes our study and compares it with the results of Legrand and Simon (1989) for the period 1868-2009. We defined four classes of geomagnetic activity but change the criteria from the classifications of (Legrand and Simon, 1989). These new criteria allow us to positively classify $80 \%$ (as opposed to $60 \%$ previously). Thus we defined: (1) quiet-days, those days with an Aa index of geomagnetic activity below $20 \mathrm{nT}$; (2) recurrent activity, the days under the control of recurrent high wind stream with an Aa index above $20 \mathrm{nT}$ without SSC; (3) shock activity, which we attribute to CMEs. We select only those days with non-recurrent SSCs and days within three days after the SSC which show enhanced Aa value above $20 \mathrm{nT}$; (4) unclear activity. This transient activity is formed by the days which do not fit in the three other classes.

The present refinement in geomagnetic activity classification constitutes a starting point towards a standard classification of solar activity using especially solar physics and solar phenomenon before their geoeffectiveness.

Acknowledgements. The authors thank all the members of LPP/CNRS/UPMC for their welcome.

The authors thank OMNI data base for providing the values of solar wind velocity and sunspot numbers.

We express many thanks to Coopération Française and Burkina Faso for their financial help. JDR was supported by NASA's Wind mission.

Many thanks to Jean-Pierre Legrand for his scientific help and advice.

Topical Editor K. Kauristie thanks C. Demetrescu and L. Svalgaard for their help in evaluating this paper.

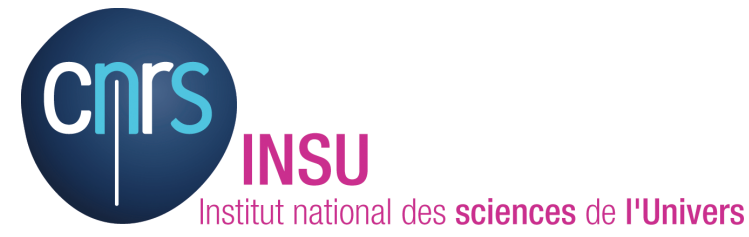

The publication of this article is financed by CNRS-INSU.

\section{References}

Gopalswamy, N., Lara, A., Yashiro, S., and Howard, R. A.: Coronal Mass Ejections and solar polarity reversal, The Astrophysical Journal, 598, L63-L66, 2003.

Legrand, J. P. and Simon, P. A.: Some solar cycle phenomena related to the geomagnetic activity from 1868 to 1980 , I. The shock events, or the interplanetary expansion of the toroidal field, Astron. Astrophys., 152, 199-204, 1985.

Legrand, J. P. and Simon, P. A.: Solar cycle and geomagnetic activity: A review for geophysicists. Part I. The contributions to geomagnetic activity of shock waves and of the solar wind, Ann. Geophys., 7, 565-578, 1989,

http://www.ann-geophys.net/7/565/1989/.

Mayaud, P. N.: Une mesure plantaire d'activité magnétique basée sur deux observatoires antipodaux, Ann. Geophys., 27, 71-73, 1971, http://www.ann-geophys.net/27/71/1971/.

Mayaud, P. N.: A hundred series of geomagnetic data, 1868-1967, IAGA Bull., 33, Zurich, p. 251, 1973.

Mayaud, P. N.: Deviation, Meaning, and Use of Geomagnetic Indices, Geophys. Mongr. Ser., vol. 22, AGU, Washington, D.C., p. $154,1980$.

Ouattara, F. and Amory-Mazaudier, C.: Solar-geomagnetic activity and Aa indices toward a Standard, J. Atmos. Solar-Terr. Phys., 71, 1736-1748, 2009. 
Ouattara, F., Amory-Mazaudier, C., Menvielle, M., Simon, P., and Legrand, J.-P.: On the long term change in the geomagnetic activity during the 20th century, Ann. Geophys., 27, 2045-2051, doi:10.5194/angeo-27-2045-2009, 2009.

Ramesh, K. B.: Coronal mass ejections and sunspots-solar cycle perspective, The Astrophysical Journal Lett., 712, L77-L80, 2010.

Richardson, I. G. and Cane, H. V.: Sources of geomagnetic activity during nearly three solar cycles (1972-2000), J. Geophys. Res., 107, 1187, doi:10.1029/2001JA000504, 2002.

Richardson, I. G., Cliver, E. W., and Cane, H. V.: Sources of geomagnetic activity over the solar cycle: Relative importance of coronal mass ejections, high-speed streams, and slow solar wind. J. Geophys. Res., 105, 18200-18213, 2000.
Simon, P. A. and Legrand, J. P.: Solar cycle and geomagnetic activity: A review for geophysicists Part II. The solar sources of geomagnetic activity and their links with sunspot cycle activity, Ann. Geophys., 7, 579-594, 1989,

http://www.ann-geophys.net/7/579/1989/.

Svalgaard, L.: Geomagnetic activity: dependence on solar wind parameters, in: Coronal holes and high speed wind streams, edited by: Zirker, J. B., Colorado Ass. Univ. Press, Boulder, 371-432, 1977. 